\title{
Análisis Histomorfométrico de la Asociación de Aceite de Oliva y Bisfosfonatos en la Remodelación Ósea Periimplantaria
}

\author{
Histomorphometric Analysis of the Association of Olive Oil \\ and Bisphosphonates on Peri-Implant Bone Remodeling
}

Cristina Escudero; Carolina Virga; Alejandra Aguzzi \& Adriana De Leonardi

ESCUDERO, C.; VIRGA, C.; AGUZZI, A. \& DE LEONARDI, A. Análisis histomorfométrico de la asociación de aceite de oliva y bisfosfonatos en la remodelación ósea periimplantaria. Int. J. Odontostomat., 11(4):481-485, 2017.

RESUMEN: Los bisfosfonatos son potentes inhibidores de la resorción ósea. El aceite de oliva $(\mathrm{O})$ presenta propiedades anti-inflamatorias y anti-oxidantes. Estudiar el efecto del tratamiento combinado de alendronato (AL) y pamidronato (PA) por vía subcutánea y de $\mathrm{O}$ vía oral sobre la regeneración tisular de cavidades óseas neoformadas.: 54 ratas macho de la línea Wistar, se dividieron en 6 grupos. Grupo control $(C)$, recibieron solución salina vía subcutánea. $G r u p o(A L)$ recibió $0,5 \mathrm{mg}$ de AL/Kg de peso corporal de por vía subcutánea. Grupo (PA) recibió de igual manera que el grupo anterior. Grupo (O) fue tratado con aceite de oliva con la dieta, $50 \mathrm{~g} / \mathrm{Kg}$ de comida. Grupo (ALO) recibió tratamiento combinado con $\mathrm{AL}$ y $\mathrm{O}$. Grupo (PAO) recibió de igual tratamiento. Los sacrificios para la toma de muestras fueron a los 15, 30, 60 y 90 días. Para los estudios histopatológicos los cortes fueron teñidos con HE y observados con microscopía óptica. Los estudios estadísticos se realizaron a través del análisis de la variancia. A los quince días las áreas de los osteocitos del grupo PA se diferencian significativamente sólo respecto al grupo AL. En cuanto a la Densidad trabecular se observa un incremento de tejido óseo en todos los grupos. O mejora cualitativamente la estructura del hueso trabecular y cortical, preservando la mineralización, el tamaño y la estructura de los cristales minerales Esto sugiere que O representa una opción terapéutica prometedora para la prevención y tratamiento de las patologías óseas.

PALABRAS CLAVE: alendronato, pamidronato, aceite de oliva, remodelación ósea.

\section{INTRODUCCIÓN}

La pérdida ósea se asocia con tales factores como la edad, la menopausia en las mujeres, el tabaquismo, el exceso de alcohol, el calcio y la vitamina $\mathrm{D}$ deficiencia, masa bajo peso y músculo, anticonvulsivante y el uso de corticosteroides, así como ciertas condiciones comórbidas, como la artritis reumatoide. A nivel mundial, se ha estimado que las fracturas causadas por la osteoporosis cuentan para aproximadamente una de cada tres entre las mujeres, y aproximadamente una de cada cinco entre los hombres mayores de 50 . Aunque los mecanismos subyacentes a la osteoporosis no se entienden completamente, hay evidencia que sugiere que el estrés oxidativo causado por especies reactivas del oxígeno (ROS) está asociado con su patogénesis (Seeman \& Delmas, 2006; Raisz, 2005; Gimble et al., 2006; Zvonic et al., 2006; Duque, 2008).
Aceite de oliva virgen se produce a partir de las primeras prensas y segundo de la fruta de oliva por el método de prensado en frío (en la que no sólo los productos químicos y una pequeña cantidad de calor se aplican) y se compone de una fracción de glicerina (que constituyen 90-99 \% de la aceituna) y una fracción no saponificable o glicerol (que componen 0,4 - 5 $\%$ de la aceituna), que contiene compuestos fenólicos. Históricamente, los efectos beneficiosos para la salud de la ingesta de aceite de oliva virgen se atribuyeron a la fracción de glicerina con su alta concentración de ácidos grasos monoinsaturados (MUFA) (en particular el ácido oleico). Sin embargo, una serie de aceites de semillas (incluyendo girasol, soja, colza etc.) que contienen altas cantidades de grasas monoinsaturadas son ineficaces en beneficiosamente la alteración de

Cátedras de Farmacología y Terapéutica A y B. Departamento de Patología Oral, Facultad de Odontología. Universidad Nacional de Córdoba,Córdoba, Argentina. 
los factores de riesgo de enfermedades crónicas. Por lo tanto, se han realizado un número considerable de investigaciones que examinan las acciones biológicas de los compuestos fenólicos del aceite de oliva en la fracción insaponificable (Vissers et al., 2004; Puel et al., 2004; Puel et al., 2006).

Los bifosfonatos, difieren en su estructura química con el pirofosfato. Éstos, presentan dos átomos de fósforo unidos entre sí por un átomo de carbono $(\mathrm{P} \mathrm{C}$ $P)$, dotando a este enlace de una mayor resistencia frente a la acción enzimática de la fosfatasa. Además, sus dos enlaces libres le confieren la posibilidad de concatenarse con cadenas laterales, lo cual, unido a la presencia de su grupo hidroxilo, determina la gran afinidad de esta molécula por los cristales de hidroxiapatita que conforman la estructura de nuestros huesos. La acción de los bifosfonatos será la de migrar y depositarse en aquellas localizaciones en las que el proceso de resorción ósea se encuentre aumentado y en donde el osteoclasto como célula activa se encuentra degradando los componentes orgánicos y minerales del hueso. La molécula de bifosfonato se deposita entre los cristales de hidroxiapatita, los cuáles serán disueltos por el osteoclasto, procediendo con ello a la endocitosis del bifosfonato. Una vez dentro, la molécula de bifosfonato se deposita en el citosol del osteoclasto, procediendo a su inactivación definitiva (Puel et al., 2006; Santamaria et al., 2010; Veena \& Prasad, 2010).

El objetivo de este trabajo fue estudiar el efecto de la administración de AL y PA por vía subcutánea y de O por vía oral la regeneración tisular de cavidades óseas neoformadas para su aplicación en el ambiente perimplantario.

\section{MATERIAL Y MÉTODO}

Preparación de las formulaciones: Se prepararon soluciones de AL y PA para ser aplicados por vía subcutánea. La fórmula farmacéutica con $A L$ se preparó con una dosificación de $0.5 \mathrm{mg} / \mathrm{Kg}$ de peso corporal, y la de PA con una dosificación de $0,6 \mathrm{mg} / \mathrm{Kg}$ de peso corporal. El O se administró junto con la dieta, $50 \mathrm{~g} /$ $\mathrm{Kg}$ de comida.

Preparación de los animales de experimentación: Cincuenta y cuatro ratas macho de la línea Wistar de peso $160 \pm 20 \mathrm{~g}$, se dividieron en 6 grupos de 9 ratas cada uno. Un grupo actuó como grupo control (C). Los animales de este grupo recibieron semanalmente 0,3 $\mathrm{ml} / 100 \mathrm{~g}$ de peso corporal de solución salina por vía subcutánea cercana a la intervención quirúrgica y se administró agua corriente de red como agua de bebida. El segundo grupo $(A L)$ se dosificó semanalmente 0,5 $\mathrm{mg}$ de $\mathrm{AL} / \mathrm{Kg}$ de peso corporal por vía subcutánea profunda en el miembro posterior izquierdo cercano a la zona quirúrgica, y en el miembro posterior derecho se le aplicó solución salina como el grupo control. El tercer grupo (PA) recibió semanalmente $0,6 \mathrm{mg}$ de $\mathrm{PA} / \mathrm{Kg}$ de peso corporal por vía subcutánea profunda en el miembro posterior izquierdo cercano a la zona quirúrgica, y en el miembro posterior derecho se suministró solución salina como el grupo control. El cuarto grupo (O), recibirá tratamiento con $\mathrm{O}$, siendo alimentados ad libitum, con una ración de $40 \mathrm{~g}$ día/rata, al que se le adicionó previamente el principio activo durante el tiempo que duró el experimento y en las áreas de las cirugías recibieron inyección subcutánea de solución salina como el grupo control. El quinto grupo de tratamiento se administró en forma combinada AL subcutáneo y $\mathrm{O}$ por vía oral en la dieta $(A L+O)$. El sexto grupo de tratamiento combinado PA subcutáneo y O por vía oral en ladieta $(\mathrm{PA}+\mathrm{O})$

Los animales se mantuvieron en bioterio en jaulas colectivas con alimento balanceado y agua de bebida ad libitum, a una temperatura de $22-26^{\circ} \mathrm{C}$, con un ciclo luz-oscuridad: $12 \mathrm{hs}-12 \mathrm{hs}$ durante el tiempo que duró el experimento. El manejo de los animales es realizado siguiendo los lineamientos del National Institute of Health $(\mathrm{NIH})$ para el uso y cuidado de animales de experimentación (Veena \& Prasad).

Al inicio del experimento los animales fueron anestesiados con una solución de ketamina/xilazina en relación $8 \mathrm{mg} / 1.28 \mathrm{mg}$ respectivamente por cada $100 \mathrm{~g}$ de peso corporal. Previa asepsia del campo quirúrgico con yodopovidona. Se realizó con bisturí Bard Parker y hoja $\mathrm{N}^{\circ} 15$ una incisión longitudinal en ambas tibias y se procedió al decolado hasta llegar a exponer el hueso. Con una fresa número 6 y a rotación manual para no producir necrosis ósea, se realizó una cavidad en la parte plana de cada tibia hasta llegar al hueso medular. Dicha cavidad no fue rellenada con ningún material y sólo será reparada por su propio coágulo. Luego de realizada las intervención quirúrgica, se recolocaron los planos en posición y se suturó la herida con hilo reabsorbible.

Los animales fueron tratados según normas universales de asepsia. Al finalizar el experimento se sometieron a eutanasia los animales mediante inyección intracardíaca de cloruro de potasio, bajo anestesia general (Carr et al., 2006). 
Se efectuaron determinaciones en los días 0,7 , 15, 30, 60, 90 administrando la solución salina, el AL y el PA cada 7 días; y en forma continua el $\mathrm{O}$ al cuarto, quinto y sexto grupo. Los sacrificios para la toma de muestras fueron a los $0,7,15,30,60$ y 90 días.

Estudio histomorfométrico: Al finalizar el experimento se extrajeron ambas tibias y fueron colocadas en frascos estériles y fijados en formaldehído al $10 \%$ para posteriormente ser descalcificadas y luego ser incluidas en parafina. Se procedió a realizar cortes histológicos a nivel de las cavidades quirúrgicas realizadas y de hueso circundante y fueron coloreados con Hematoxilina/Eosina (H/E) y método Tricrómico de Masson; para el análisis histopatológico e histomorfométrico correspondiente.

Método de medición: mediante el software para morfometría Image Pro Plus, se delimitaron las áreas ocupadas por hueso trabecular y se calcularon los porcentajes de respecto al área total de la imagen. Se analizaron al menos 5 imágenes de dos zonas específicas: 5 en la epífisis y 5 en la diáfisis. Este procedimiento se llevó a cabo en cada grupo y etapa $(15,30,60$ y 90 días) (NHI Guide for the care and use for Laboratory Animals, 1985; Rigalli \& Di Loreto, 2009).

Estudios estadísticos: La comparación de los datos se realizará por análisis de la varianza a dos criterios de clasificación (tratamientos: $\mathrm{C}, \mathrm{AL}, \mathrm{PA}, \mathrm{O}, \mathrm{AL}+\mathrm{O}$,
$\mathrm{PA}+\mathrm{O}$, y tiempos de tratamiento: 3060 y 90 días). Se utilizarán tests paramétricos o no en función que los resultados obtenidos permitan o no verificar distribución normal de las variables utilizadas (Test de Kolmorow Smirnov). Se considerarán diferencias significativas si $p>0,05$. Este estudio estadístico planteado además permitirá determinar si existe asociación entre los efectos de los tratamientos y el tiempo. Para el análisis de datos, construcción de gráficas y análisis estadísticos se utilizará el software (GraphPad Prism 2.0. GraphPad Software, San Diego, CA USA).

\section{RESULTADOS}

Histológicamente se puede observar que en la etapa inicial (o días), las áreas de los osteocitos no difieren significativamente entre los grupos analizados. A los siete días las áreas de los osteocitos del grupo C difieren significativamente respecto al resto de los grupos analizados. A los quince días las áreas de los osteocitos del grupo PA se diferencian significativamente sólo respecto al grupo AL, con un aumento de la calidad ósea a través aumento del grosor de las trabéculas y algunas anastomósis entre ellas. Se puede observar un mayor número de osteoblastos revistiendo las trabéculas (Fig. 1) A los 30,60 y 90 días las áreas de los osteocitos no difieren significativamente entre los grupos analizados.

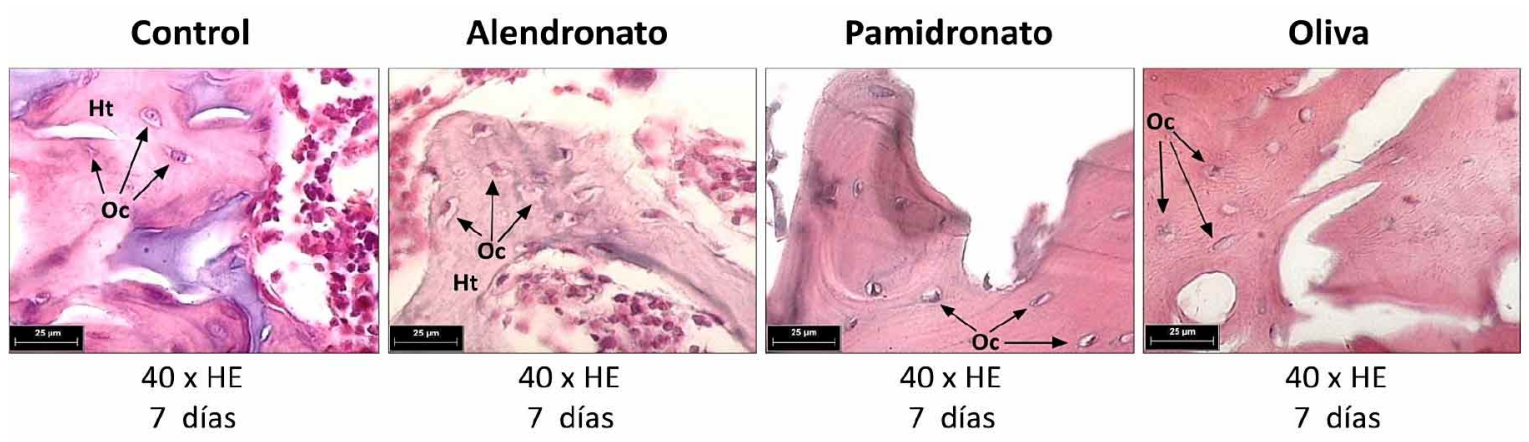

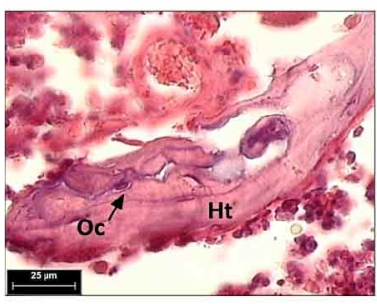

$40 \times \mathrm{HE}$

15 días

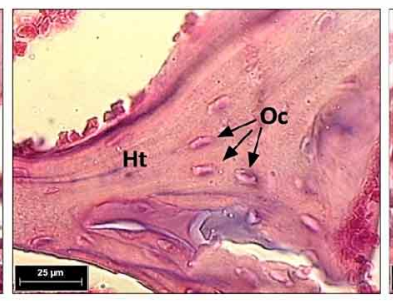

$40 \times \mathrm{HE}$

15 días

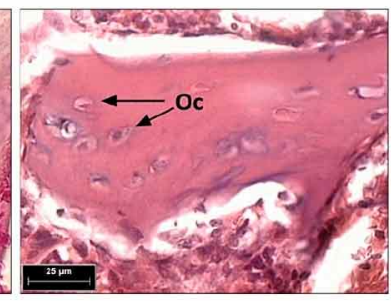

$40 \times \mathrm{HE}$

15 días

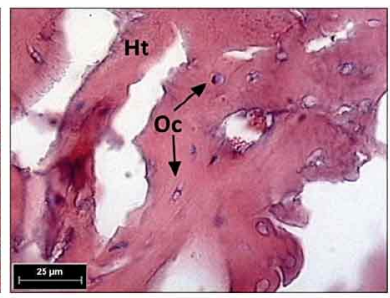

$40 \times \mathrm{HE}$

15 días

Fig. 1. Cortes histológicos a nivel de las cavidades quirúrgicas realizadas y de hueso circundante. 
En cuanto a la Densidad trabecular se observa un incremento de tejido óseo en todos los grupos, en especial en las últimas etapas (60 y 90 días), pero aunque Oliva registró valores por encima que el resto, estas diferencias no resultaron estadísticamente significativas $(p>0.05)$ (Tabla I).

\section{DISCUSIÓN Y CONCLUSIONES}

Tabla I. Densidad trabecular (\%)

\begin{tabular}{ccccc}
\hline Días & Control & ALO & PAO & Oliva \\
\hline $\mathbf{0}$ & $44 \%$ & $41 \%$ & $47 \%$ & $48 \%$ \\
$\mathbf{7}$ & $47 \%$ & $35 \%$ & $46 \%$ & $58 \%$ \\
$\mathbf{1 5}$ & $51 \%$ & $44 \%$ & $51 \%$ & $62 \%$ \\
$\mathbf{3 0}$ & $53 \%$ & $57 \%$ & $54 \%$ & $68 \%$ \\
$\mathbf{6 0}$ & $54 \%$ & $68 \%$ & $74 \%$ & $81 \%$ \\
$\mathbf{9 0}$ & $56 \%$ & $77 \%$ & $79 \%$ & $83 \%$ \\
\hline
\end{tabular}

La dieta mediterránea se asocia con una menor incidencia de aterosclerosis, enfermedades cardiovasculares, enfermedades neurodegenerativas y ciertos tipos de cáncer. Los beneficios para la salud aparentes han sido parcialmente atribuidos al consumo de aceite de oliva virgen por las poblaciones del Mediterráneo. Mucha investigación se ha centrado en los compuestos fenólicos biológicamente activos presentes de forma natural en los aceites de oliva vírgenes para ayudar en la explicación de reducción de la mortalidad y la morbilidad que experimentan las personas que consumen una dieta mediterránea tradicional. Estudios (humano, animal, in vivo e in vitro) han demostrado que los compuestos fenólicos del aceite de oliva tienen efectos positivos en ciertos parámetros fisiológicos, como las lipoproteínas del plasma, el daño oxidativo, los marcadores de inflamación, plaquetas y función celular, actividad antimicrobiana y la salud ósea (Kasdallah-Grissa et al., 2008; Cicero et al., 2008; Puel et al., 2008; Kulak \& Dempster 2010).

Santiago-Mora et al. (2011) en sus resultados muestran un aumento en la diferenciación de osteoblastos y una disminución en la diferenciación de adipocitos cuando hay oleuropeína en los medios de cultivo. La expresión de genes de marcadores de la osteoblastogénesis, colágeno tipo I, osteocalcina y la fosfatasa alcalina, era mayor en las células tratadas con la oleuropeína inducida por osteoblastos. Además, la actividad de la fosfatasa alcalina y de la mineralización de la matriz extracelular fue mayor cuando la oleuropeína estaba presente en los medios de cultivo. La oleuropeína inducida por los adipocitos para producir una disminución en la expresión de los genes implicados en la adipogénesis.

Habauzit \& Horcajada (2008) demostraron que las investigaciones proporcionan alguna evidencia de un efecto positivo de algunos fitoquímicos en el metabolismo óseo están acumulando, pero se necesitan más estudios, ensayos clínicos, sobre todo para explorar las diferentes actividades biológicas que ofrece este tipo de compuestos. De todos modos, se puede postular que el aumento de consumo de alimentos derivados de plantas, especialmente frutas y verduras, puede ser positivo en la prevención de la osteoporosis.

Nuestra investigación apoya el efecto beneficioso del aceite de oliva relatado por los autores anteriores, ya que histológicamente se pudo observar un aumento de la calidad ósea a través aumento del grosor de las trabéculas y algunas anastomosis entre ellas; como así también un mayor número de osteoblastos revistiendo las trabéculas.

De la misma manera, nuestro estudio histomorfométrico reveló que el aceite de oliva produce un incremento de tejido óseo en todos los tiempos experimentales, con aumento de la densidad trabecular, sobre todo en tiempos largos (60 y 90 días).

Podemos concluir que en base a esta investigación se considera al $\mathrm{O}$ de acción dual capaz de disociar el remodelado óseo reequilibrándolo hacia la formación de hueso, actúa a nivel de todos y cada uno de los determinantes de la resistencia ósea, mejorando cualitativamente la estructura del hueso trabecular y cortical y preservando la mineralización, el tamaño y la estructura de los cristales minerales incluso a dosis elevadas. De esta manera, O mejora significativamente las propiedades biomecánicas del hueso.

ESCUDERO, C.; VIRGA, C.; AGUZZI, A. \& DE LEONARDI, A. Histomorphometric analysis of the association of olive oil and bisphosphonates on peri-implant bone remodeling. Int. J. Odontostomat., 11(4):481-485, 2017.

ABSTRACT: Bisphosphonates are potent inhibitors of bone resorption. Olive oil $(\mathrm{O})$ has anti-inflammatory and anti-oxidant properties. To study the effect of combined treatment with alendronate $(A L)$ and pamidronate (PA) subcutaneously or orally, and on tissue regeneration of newly formed bone cavities, we divided 54 male Wistar rats into 6 
groups. Control group $(\mathrm{C})$ received saline subcutaneously. Group $(A L)$ received $0.5 \mathrm{mg}$ of $\mathrm{AL} / \mathrm{kg}$ body weight subcutaneously. Group (PA) received the same as the previous group. (O) was treated with olive oil diet, $50 \mathrm{~g} / \mathrm{kg}$ of food. Group (ALO) received combined treatment with $A L$ and O. Group (PAO) received the same treatment. The animals were euthanized for sampling at 15, 30, 60 and 90 days. For histopathology sections were stained with $\mathrm{HE}$ and observed these with light microscopy. Statistical studies were performed by analysis of variance. Fifteen days osteocytes areas of the PA group were significantly different only for the $\mathrm{AL}$ group. As the density increased trabecular bone tissue was observed in all groups. O qualitatively improved the structure of trabecular and cortical bone mineralization while preserving the size and structure of the mineral crystals. This suggests that $O$ represents a promising therapeutic option for prevention and treatment of bone diseases.

KEY WORDS: alendronate, pamidronate, olive oil, bone remodeling.

\section{REFERENCIAS BIBLIOGRÁFICAS}

Carr, A. J.; Thompson, P. W. \& Cooper, C. Factors associated with adherence and persistence to bisphosphonate therapy in osteoporosis: a cross-sectional survey. Osteoporos. Int., 17(11):1638-44, 2006.

Cicero, A. F.; Nascetti, S.; López-Sabater, M. C.; Elosua, R.; Salonen, J. T.; Nyyssönen, K.; Poulsen, H. E.; Zunft, H. J.; Kiesewetter, H.; de la Torre, K.; Covas, M. I.; Kaikkonen, J.; Mursu, J.; Koenbick, C.; Bäumler, H.; Gaddi, A. V. \& EUROLIVE Study Group. Changes in LDL fatty acid composition as a response to olive oil treatment are inversely related to lipid oxidative damage: The EUROLIVE study. J. Am. Coll. Nutr., 27(2):314-20, 2008.

Duque, G. Bone and fat connection in aging bone. Curr. Opin. Rheumatol., 20(4):429-34, 2008.

Gimble, J. M.; Zvonic, S.; Floyd, Z. E.; Kassem, M. \& Nuttall, M. E. Playing with bone and fat. J. Cell. Biochem., 98(2):251-66, 2006.

Habauzit, V. \& Horcajada, M. N. Phenolic phytochemicals and bone. Phytochem. Rev., 7(2):313-44, 2008.

Kasdallah-Grissa, A.; Nakbi, A.; Koubaa, N.; El-Fazaâ, S.; Gharbi, N.; Kamoun, A. \& Hammami, M. Dietary virgin olive oil protects against lipid peroxidation and improves antioxidant status in the liver of rats chronically exposed to ethanol. Nutr. Res., 28(7):472-9, 2008.

Kulak, C. A. \& Dempster, D. W. Bone histomorphometry: a concise review for endocrinologists and clinicians. Arq. Bras. Endocrinol. Metabol., 54(2):87-98, 2010.

$\mathrm{NHI}$ Guide for the care and use for Laboratory Animals. Departament of Health and Human Service, Public Health Service, National Institutes of Health Publication Nro. 85.23, 1985.

Puel, C.; Mardon, J.; Agalias, A.; Davicco, M. J.; Lebecque, P.; Mazur, A.; Horcajada, M. N.; Skaltsounis, A. L. \&
Coxam, V. Major phenolic compounds in olive oil modulate bone loss in an ovariectomy/inflammation experimental model. J. Agric. Food Chem., 56(20):9417-22, 2008.

Puel, C.; Mathey, J.; Agalias, A.; Kati-Coulibaly, S.; Mardon, J.; Obled, C.; Davicco, M. J.; Lebecque, P.; Horcajada, M. N.; Skaltsounis, A. L. \& Coxam, V. Dose-response study of effect of oleuropein, an olive oil polyphenol, in an ovariectomy/inflammation experimental model of bone loss in the rat. Clin. Nutr., 25(5):859-68, 2006.

Puel, C.; Quintin, A.; Agalias, A.; Mathey, J.; Obled, C.; Mazur, A.; Davicco, M. J.; Lebecque, P.; Skaltsounis, A. L. \& Coxam, V. Olive oil and its main phenolic micronutrient (oleuropein) prevent inflammation-induced bone loss in the ovariectomised rat. Br. J. Nutr., 92(1):119-27, 2004.

Raisz, L. G. Pathogenesis of osteoporosis: concepts, conflicts, and prospects. J. Clin. Invest., 115(12):331825, 2005.

Rigalli, A. \& Di Loreto, V. Experimental Surgical Models in the Laboratory Rat. Boca Ratón, CRC Press, 2009.

Santamaria, M. Jr.; Fracalossi, A. C.; Consolaro, M. F. \& Consolaro, A. Influence of bisphosphonates on alveolar bone density: a histomorphometric analysis. Braz. Oral Res., 24(3):309-15, 2010.

Santiago-Mora, R.; Casado-Díaz, A.; De Castro, M. D. \& Quesada-Gómez, J. M. Oleuropein enhances osteoblastogenesis and inhibits adipogenesis: the effect on differentiation in stem cells derived from bone marrow. Osteoporos. Int., 22(2):675-84, 2011.

Seeman, E. \& Delmas, P. D. Bone quality--the material and structural basis of bone strength and fragility. N. Engl. J. Med., 354(21):2250-61, 2006.

Veena, H. R. \& Prasad, D. Evaluation of an aminobisphosphonate (alendronate) in the management of periodontal osseous defects. J. Indian Soc. Periodontol., 14(1):40-5, 2010.

Vissers, M. N.; Zock, P. L. \& Katan, M. B. Bioavailability and antioxidant effects of olive oil phenols in humans: a review. Eur. J. Clin. Nutr., 58(6):955-65, 2004.

Dirección para correspondencia:

Dra. Alejandra Aguzzi

Haya de la Torre s/n

Facultad de Odontología

Ciudad Universitaria

Córdoba

ARGENTINA

Email: aleceagu@yahoo.com.ar 